\title{
Inspirée face aux défis : l'expérience d'une professeure non autochtone en lien à l'intégration des perspectives autochtones dans la formation des enseignants en contexte minoritaire francophone manitobain
}

\author{
Laura Sims
}

Volume 31, numéro 1, 2019

L’autochtonisation pour préparer un avenir commun

URI : https://id.erudit.org/iderudit/1059127ar

DOI : https://doi.org/10.7202/1059127ar

\section{Aller au sommaire du numéro}

Éditeur(s)

Presses universitaires de Saint-Boniface (PUSB)

ISSN

0843-9559 (imprimé)

1916-7792 (numérique)

Découvrir la revue

Citer cet article

Sims, L. (2019). Inspirée face aux défis : l'expérience d'une professeure non autochtone en lien à l'intégration des perspectives autochtones dans la formation des enseignants en contexte minoritaire francophone manitobain. Cahiers franco-canadiens de l'Ouest, 31(1), 89-108.

https://doi.org/10.7202/1059127ar
Résumé de l'article

L’intégration des perspectives autochtones en éducation est importante lorsqu'on entreprend d'aborder les inégalités et les injustices subies depuis longtemps par les Autochtones à cause de stéréotypes négatifs et du racisme. Comme pédagogues non autochtones et alliés, nous avons un rôle à jouer. Par contre, appuyer le processus avec dignité et respect n'est pas toujours évident. En effet, cette démarche amène un questionnement sur le sens réel de l'intégration des perspectives autochtones et sur les stratégies d'enseignement à déployer pour y parvenir: que devrais-je faire sur le plan pédagogique? Par où commencer? Le but de cet article est de partager mon expérience comme facilitatrice de l'apprentissage et des connaissances autochtones lors de l'enseignement du cours Perspectives autochtones en contexte scolaire au baccalauréat en éducation, à l'Université de Saint-Boniface. Contextualisée par la littérature pertinente, cette exploration traite de la façon dont j'ai abordé et mis en oeuvre le cours depuis les six dernières années. Plus précisément, j'explore mon rôle en tant que pédagogue, mes objectifs, mon approche pour construire une communauté d'apprentissage enrichissante. Je discute de la façon dont le cours a été mis en oeuvre de manière participative. Des considérations pour l'enseignement de ce cours dans un contexte francophone minoritaire manitobain sont explorées.
Tous droits réservés (C Centre d'études franco-canadiennes de l'Ouest (CEFCO) et Presses universitaires de Saint-Boniface (PUSB), 2019
Ce document est protégé par la loi sur le droit d'auteur. L'utilisation des services d'Érudit (y compris la reproduction) est assujettie à sa politique d'utilisation que vous pouvez consulter en ligne.

https://apropos.erudit.org/fr/usagers/politique-dutilisation/ 


\title{
Inspirée face aux défis: l'expérience d'une professeure non autochtone en lien à l'intégration des perspectives autochtones dans la formation des enseignants en contexte minoritaire francophone manitobain*
}

\author{
Laura SIMS
}

Université de Saint-Boniface

\begin{abstract}
RÉSUMÉ
L'intégration des perspectives autochtones en éducation est importante lorsqu'on entreprend d'aborder les inégalités et les injustices subies depuis longtemps par les Autochtones à cause de stéréotypes négatifs et du racisme. Comme pédagogues non autochtones et alliés, nous avons un rôle à jouer. Par contre, appuyer le processus avec dignité et respect n'est pas toujours évident. En effet, cette démarche amène un questionnement sur le sens réel de l'intégration des perspectives autochtones et sur les stratégies d'enseignement à déployer pour y parvenir: que devrais-je faire sur le plan pédagogique? Par où commencer? Le but de cet article est de partager mon expérience comme facilitatrice de l'apprentissage et des connaissances autochtones lors de l'enseignement $\mathrm{du}$ cours Perspectives autochtones en contexte scolaire au baccalauréat en éducation, à l'Université de SaintBoniface. Contextualisée par la littérature pertinente, cette exploration traite de la façon dont j'ai abordé et mis en œuvre le cours depuis les six dernières années. Plus précisément, j'explore mon rôle en tant que pédagogue, mes objectifs, mon approche pour construire une
\end{abstract}

L'auteure exprime sa reconnaissance aux membres de la communauté autochtone qui ont contribué à faire de ce cours une expérience d'apprentissage riche pour ses étudiants et elle. L'auteure remercie les évaluateurs anonymes pour leurs précieux commentaires et conseils sur l'amélioration de ce document. Veuillez noter que le masculin est utilisé afin d'alléger le texte 
communauté d'apprentissage enrichissante. Je discute de la façon dont le cours a été mis en œuvre de manière participative. Des considérations pour l'enseignement de ce cours dans un contexte francophone minoritaire manitobain sont explorées.

\section{Introduction}

Parfois, les circonstances nous obligent à sortir de notre zone de confort. Il y a sept ans (en 2012), j'ai été invitée à enseigner le cours Perspectives autochtones en contexte scolaire aux étudiants de première année du baccalauréat en éducation de l'Université de Saint-Boniface, à Winnipeg au Manitoba. Ce cours, offert depuis 2008 au Manitoba, est le résultat de plus de dix années de consultations, de recherches et d'élaboration de programmes d'études dans le contexte où une population autochtone en plein essor présente toujours un faible taux d'obtention de diplôme (Association canadienne des doyens et doyennes d'éducation, 2010; Gouvernement du Manitoba, 2007; Manitoba Association of School Superintendents, 2013). Enseigner ce cours était un honneur, une merveilleuse occasion unique et une responsabilité importante; cela représentait également une tâche assez intimidante. En tant que professeure non autochtone, je me demandais si j'avais la légitimité d'enseigner ce cours car, au Manitoba, celui-ci avait la réputation d'être difficile à mettre en œuvre ${ }^{1}$. Ainsi, après de nombreuses consultations auprès de mes collègues autochtones du Manitoba, particulièrement auprès de ceux des universités anglophones, j'ai accepté d'enseigner ce cours. Ces collègues, et les autres membres de la communauté autochtone qui ont partagé leurs connaissances avec moi et les étudiants inscrits au cours en question, ont contribué de manière considérable à son succès: ils sont de loin mon plus grand cadeau. Ils étaient accueillants, encourageants, avaient confiance en mes capacités et ont accepté de m'accompagner tout au long du processus. En tant que professeure et Canadienne, je peux dire honnêtement que cette expérience d'enseignement a été profondément enrichissante, particulièrement en ce qui a trait à la compréhension des responsabilités, au rapprochement des communautés, à l'espoir et à la création de possibilités pour l'avenir. Tout en appréciant l'importance et la valeur de ce cours dans le processus de transformation de la société canadienne 
par l'éducation pour qu'elle soit plus équitable, respectueuse et inclusive, j'étais nerveuse et j'avais un peu peur. Souvent, lorsqu'il s'agit d'aborder des sujets potentiellement sensibles, il est difficile de savoir par où commencer et, par conséquent, nous sommes paralysés par cette incertitude.

Avant même d'enseigner le cours Perspectives autochtones en contexte scolaire, il y a environ neuf ans, je me suis rendu compte que j'avais beaucoup à apprendre comme citoyenne, Winnipegoise, Canadienne et pédagogue quant à notre histoire coloniale, à ses répercussions sur notre société actuelle et surtout sur les peuples autochtones. Du même coup, je prenais conscience des implications de ces enjeux par rapport à l'éducation publique, et plus précisément en lien avec ma responsabilité dans la formation des futurs enseignants. À cette époque, j'avais commencé à collaborer avec le Groupe de recherche sur l'éducation autochtone du Manitoba Education Research Network (MERN - Réseau de recherche en éducation du Manitoba) pour promouvoir l'éducation autochtone. En tant que pédagogue canadienne non autochtone et alliée dans ce processus, il m'a toujours été un défi de soutenir concrètement, avec dignité et respect, les perspectives autochtones en éducation en raison de mes nombreux questionnements (c.-à-d. ce que signifie réellement la notion d'intégration des perspectives autochtones en éducation). Que devrais-je faire dans ma pédagogie? Par où commencer?). Le but de cet article est de partager mon expérience sur la façon dont j'ai négocié le rôle de facilitatrice de l'apprentissage et des connaissances autochtones lors de l'enseignement du cours Perspectives autochtones en contexte scolaire. Plusieurs idées dans cet article ont déjà été explorées par Sims (2015). Le présent article vise à élaborer davantage certaines idées, à mettre l'information à jour dans le contexte manitobain qui évolue rapidement, à explorer et à considérer certains enjeux particuliers à l'enseignement en contexte francophone minoritaire. Cet article contribue à la discussion sur la façon dont la théorie pourrait être mieux mise en pratique (Beeman et Sims, sous presse; Block et al., 2016; Deer, 2013; Kanu 2005, 2011; St. Denis, 2007). Il est également destiné à aider d'autres pédagogues, en particulier non autochtones, à réfléchir à leurs rôles et responsabilités potentiels en matière d'éducation autochtone. 


\section{Le contexte de l'élaboration du cours}

Il existe de nombreuses raisons pour lesquelles il est important d'intégrer les perspectives autochtones en éducation, dont la nécessité de lutter contre les stéréotypes négatifs et le racisme envers les peuples autochtones au Canada (Comack et al., 2009; Macdonald, 2015; Sinclair, 2016; St.-Denis, 2007). Afin d'éliminer cesinjustices et d'autres, il estnécessaire qu'un système d'éducation soit réellement inclusif et «réceptif aux défis de la diversitéet dela différence dans la scolarité publique» [traduction libre] (Kanu, 2011, p. 49). Kanu (2005) soutient que le but de l'intégration est de s'attaquer aux «inégalités qui ont engendré pendant des générations des inégalités économiques, sociales et éducatives pour les peuples autochtones» [traduction libre] (p. 66). Cette intégration efficace des perspectives autochtones dans la pratique éducative au sein des écoles présente certains défis uniques à relever. Un héritage historique entraîne un malaise, de la tension et de la culpabilité au moment de discuter d'enjeux liés aux questions autochtones et à l'intégration possible des perspectives autochtones dans le programme scolaire (Deer, 2013). Souvent, les enseignants éprouvent une appréhension face à leur manque de connaissances (Kanu, 2005). De plus, les enseignants non autochtones manquent de confiance en leurs connaissances sur les perspectives et le contenu du sujet et craignent que celles-ci soient perçues comme illégitimes ou non authentiques (Kanu, 2011). En dépit de ces défis, St.-Denis (2007) et Sinclair (2016) affirment que nous devons reconnaître l'importance de discuter de ces questions même si cela mène à aborder des sujets sensibles.

Dans son enseignement aux futurs enseignants de l'intégration des perspectives autochtones, Kanu (2011) insiste sur la nécessité de créer une communauté qui soit encourageante et stimulante, et au sein de laquelle les hypothèses de base peuvent être critiquées. Kanu (idem) encourage les pédagogues à remettre en question leurs propres idées préconçues afin de permettre aux élèves de faire de même. En effet, l'étude de Kanu (2005) a révélé que l'évolution des attitudes des enseignants est essentielle si nous voulons réussir à intégrer les perspectives autochtones en éducation. Elle nous invite à voir le curriculum non seulement comme un cours à l'étude, mais aussi comme une expérience personnelle, ce qu'elle appelle currere. L'auteure 
nous invite à voir le curriculum comme une conversation dans un but de compréhension, afin de permettre le partage de pouvoir dans le processus d'apprentissage, de reconnaître les riches connaissances et expériences que les étudiants ainsi que les membres de la communauté apportent à la discussion. Elle soutient que notre objectif à l'université devrait être d'aider les futurs enseignants à renforcer leurs capacités (c.-àd. leur connaissance du sujet et les stratégies d'enseignement pertinentes) et de créer un réseau de ressources pour accroître leur sentiment d'efficacité dans l'intégration des perspectives autochtones. Face au défi d'aborder l'héritage de la colonisation, Kanu (2011) propose de présenter:

des connaissances qui équilibrent les forces des peuples autochtones avec les problèmes auxquels ils sont confrontés et une analyse des racines sociales, économiques et politiques de ces problèmes» [traduction libre] (p. 215).

Cela donnerait aux apprenants (c.-à-d. aux futurs enseignants) une image plus précise de la résilience des peuples autochtones. En effet, Kanu (idem) a constaté que la présentation de «contrehistoires» ${ }^{2}$ favorisait le développement de compétences cognitives plus élevées et une meilleure compréhension des enjeux autochtones, permettant ainsi de renverser des idées stéréotypées négatives. En ce qui concerne les méthodes appropriées pour intégrer les perspectives autochtones à l'enseignement, Kanu $(2005,2011)$ insiste sur l'innovation, par le recours à des méthodes telles que: le storytelling (les histoires de cas), inviter des conférenciers, faire des excursions, des apprentissages gradués, des cercles de discussion et promouvoir la rédaction des journaux de bord et la réalisation des projets en petits groupes. St.-Denis (2007) encourage également l'intégration des traditions et des pratiques culturelles. À partir de mon expérience d'enseignement et de recherche, j'avais élaboré intuitivement plusieurs de ces principes avant d'enseigner le cours; le travail de Kanu apporte donc une validation et fournit un contexte théorique à l'approche que j'avais préconisée.

Ainsi, avec humilité et une ouverture d'esprit, mon objectif était d'intégrer des voix authentiques et d'identifier des messages clés afin de faire de ce cours un parcours d'apprentissage positif. J'ai beaucoup réfléchi à l'environnement d'apprentissage et aux relations, ainsi qu'à la manière dont 
j'allais atteindre mes objectifs. Les évaluations formelles et informelles des cours, les commentaires des étudiants, leurs journaux de bord et mes observations personnelles des attitudes et des niveaux de participation aux activités (au cours des six dernières années et à travers les douze derniers groupes) ont confirmé que ces objectifs ont été atteints. Une récente entrevue accordée au Téléjournal Manitoba par Erica (le 8 novembre 2018), une étudiante qui a suivi le cours l'année dernière, exemplifie l'impact de ce processus d'apprentissage. Selon moi, cet exemple illustre une prise de conscience menant au praxis (voir l'entrevue à: https://ici.radio-canada.ca/tele/le-telejournalmanitoba/site/segments/reportage/94178/etudiants-universitede-saintboniface-drag-the-red). Dans l'entrevue, Erica décrit comment, dans le cours, nous avons eu plusieurs invités qui ont parlé de différentes initiatives communautaires. Erica a été profondément émue et inspirée par la présentation de Mitch Bourbonnière qui travaille beaucoup avec des jeunes à risques (http://www.mitchbourbonniere.com/). Elle et d'autres lui ont demandé comment ils pourraient aider. À la suite de cette présentation, en collaboration avec d'autres membres de sa cohorte, ils ont fait une collecte de fonds pour faire des trousses de base pour Mama bear clan. Cette année (2018-2019), encore en demandant l'avis de Bourbonnière, ils ont fait une collecte de fonds pour Drag the red. À la fin de l'entretien, la journaliste demande à Erica qui devrait contribuer à cette initiative. Sa réponse est «tout le monde», car c'est une situation qui nous touche tous. Pour moi, cet exemple me montre que les étudiants ont bien compris les objectifs que je tente de communiquer tout au long du cours: je vois mes étudiants ouverts à s'éduquer sur les enjeux qui affectent notre communauté, en particulier les peuples autochtones, et que ces questions devraient être des priorités pour nous tous. Je constate que mes étudiants sont encouragés d'agir, qu'avant d'agir, ils demandent l'avis des membres de la communauté autochtone, et que leur engagement vis-à-vis des enjeux et de la communauté autochtone est maintenu. Ceci me donne beaucoup d'espoir.

\section{Mon rôle dans le processus de l'apprentissage}

En enseignant ce cours en tant que professeure en milieu universitaire, je ne me considérais pas comme une experte et ne voulais pas prétendre que la recherche qui 
vise à étudier, à comprendre les enjeux, les perspectives, les cultures, les connaissances, les façons d'être et de faire des autochtones et tout ce que cela comprend dans sa diversité et sa richesse était mon domaine d'expertise. Pourtant, j'espérais pouvoir contribuer, je situais davantage mon rôle comme une facilitatrice des connaissances tout en étant en mesure de justifier adéquatement le bien-fondé de l'intégration des perspectives autochtones en éducation. Je me sentais capable de créer des espaces sécurisants pour les aînés autochtones et les invités afin qu'ils puissent partager leurs histoires et orienter les étudiants dans des discussions critiques pouvant les mener vers une meilleure compréhension du sujet et des enjeux. Mon rôle était donc celui d'accompagnatrice des étudiants dans leur cheminement autoréflexif et introspectif face à notre héritage colonial, en vue de leur permettre d'acquérir les compétences nécessaires pour intégrer efficacement et avec confiance les perspectives autochtones dans leur approche pédagogique. Mon point de vue est que nous commençons là où nous sommes, et nous apportons ce que nous pouvons; il faut utiliser nos forces pour faire de notre mieux. Comme le soulève St.-Denis (2007), en tant quacteurs de l'éducation, nous faisons tous et toutes partie de la solution; nous devons dévoiler le racisme dont nous avons hérité.

\section{Les objectifs du cours}

Comme les sociétés canadienne et manitobaine ont évolué depuis dix ans par rapport aux enjeux autochtones, les objectifs de ce cours Perspectives autochtones en contexte scolaire (un cours de trois crédits) ont aussi évolué. Selon les plans de cours de l'Université de Saint-Boniface (2008 - 2012), le cours visait surtout à sensibiliser les étudiants à la diversité culturelle autochtone afin qu'ils puissent bien intégrer les perspectives autochtones à leur enseignement. De plus, il avait pour but de favoriser le développement de compétences professionnelles relatives à l'enseignement dans un environnement autochtone. Quand j'ai pris la responsabilité du cours, mes objectifs étaient et continuent d'être: inspirer, sensibiliser, ouvrir les esprits, enseigner le respect, partager des histoires et promouvoir la réflexion critique. Maintenant, de plus en plus, je me rends compte de l'importance d'apprendre à vivre la réconciliation, d'apprendre à s'occuper les uns des autres (les êtres humains 
ainsi que les êtres autres-que-humains), et de la Terre. Je voulais offrir un cours où les étudiants seraient assez courageux, et en mesure de développer une posture critique, pour faire face à notre héritage colonial et où ils partiraient inspirés et en mesure d'intégrer les pédagogies et le contenu autochtones dans leur propre pratique d'enseignement. Pour ce faire, il était important de créer une communauté d'apprenants qui soit encourageante, honnête, confiante, authentique et sans jugement. Consciente des limites de temps à passer avec les étudiants, je voulais fournir les outils et laisser libre cours au désir, afin que l'étudiant puisse continuer à explorer les défis et les possibilités par lui-même.

\section{L'identification des forces}

Avec ces objectifs en tête, j'ai identifié mes propres forces et celles de mon institution. En ce qui concerne mes points forts par rapport au cours, ma recherche, en termes très généraux, m’a permis d'explorer, de tester et d'évaluer la façon de créer des forums d'apprentissage où participent divers acteurs de la société qui permettent une transition vers une société plus viable, équitable et écologique (voir Sims, 2017; Sims et Falkenberg, 2013; Sims et Sinclair, 2008). Mon enseignement a souvent porté sur des questions de diversité, d'équité et de justice sociale. Mon approche était participative, critique et interdisciplinaire. Les leçons tirées de ces expériences ont contribué à la création d'un environnement d'apprentissage stimulant, au raffinement de mon approche pédagogique et à ma compréhension des apprentissages résultants de la participation des étudiants au cours.

De nombreux facteurs liés à l'établissement universitaire où j'enseigne ont été bénéfiques à la mise en œuvre de ce cours. Pour commencer, les cours étaient d'une durée de deux heures, deux fois par semaine. Jusqu'à récemment, la cohorte se composait de deux classes d'environ 20 étudiants chacune. Cette configuration de classes relativement petites et de créneaux horaires de classe plus longs était bénéfique, car elle nous permettait de discuter des sujets de manière plus approfondie (p. ex. des sujets tels que: les pensionnats autochtones, processus et impacts de la colonisation, relation des traités en contexte historique et contemporain, la résilience). Le processus d'apprentissage relié à ce cours pouvait être très émotif pour les étudiants, et avec des classes plus petites, cela 
m'a permis d'être plus attentive à leurs besoins et de les soutenir tout au long de leur démarche. Récemment, une cohorte était beaucoup plus nombreuse. Malgré ce changement, nous avons quand même réussi à avoir des discussions très riches, bien que le temps pour avoir des interactions de qualité avec tous les étudiants fût limité. Ce constat est inquiétant. De plus, par le biais de leurs travaux, j'invitais les étudiants à être honnêtes et à pousser leurs réflexions. Comme j'insiste pour être la seule personne responsable d'évaluer les travaux afin de pouvoir donner une rétroaction significative, cela devenait un défi en raison du nombre élevé d'étudiants de certaines cohortes.

Ensuite, j'ai demandé que le cours soit offert à la session d'hiver, après avoir enseigné à tous les étudiants un cours d'introduction à la diversité culturelle en éducation. Cela m'a permis, entre septembre et décembre, de présenter les concepts de préjugés et de discrimination, et de sensibiliser les étudiants à l'importance de l'inclusion et de la tolérance en éducation. Cela m'a aussi permis d'amorcer le processus d'établissement d'une relation de confiance avec les étudiants (et les uns avec les autres) avant de discuter du sujet des perspectives autochtones. De façon générale, je suis portée à croire que ces facteurs ont grandement contribué au succès du cours.

\section{La planification du cours}

Lors de la planification initiale du cours, j'ai impliqué les étudiants dans le processus, en expliquant la raison pour laquelle les travaux étaient structurés comme ils l'étaient (c.-àd. quels étaient les grands thèmes, les conférenciers potentiels, les activités culturelles, la nature des travaux) afin d'obtenir leurs rétroactions. De manière significative, cela a eu pour effet d'enthousiasmer les étudiants à propos du cours et de ce qu'ils allaient apprendre. À différents moments avant et pendant le cours, les étudiants ont été invités à participer à la prise de décisions sur les thèmes qu'ils souhaitaient approfondir. J'ai considéré le cours comme une responsabilité collective. Par exemple, avec plusieurs cohortes, nous avons décidé ensemble de visiter le Manitoba Indigenous Cultural Education Centre Inc. ${ }^{3}$ pour voir des exemples de ressources éducatives non formelles et formelles qui existent pour et dans la communauté. Pour la plupart des étudiants, c'était une première visite au sein de cet organisme et, pour certains, leur première visite dans 
la communauté de Point Douglas ${ }^{4}$. Tout au long de la mise en œuvre du cours, les étudiants ont été invités à fournir des commentaires constructifs sur les activités et, à la fin, à évaluer le cours. En intégrant l'apport et la participation des étudiants au processus de planification et d'évaluation, j'ai voulu modéliser l'approche quej'essayais d'enseigner (être flexible pour répondre aux besoins et aux intérêts des apprenants, partager le pouvoir décisionnel) (Alberta Education, 2005; Anderson et al., 2017; Éducation et Formation Manitoba, 2017; Sims, 2012, 2017). À cet effet, le cours est différent chaque année, taillé selon les intérêts et les besoins des étudiants. Certainement, cette approche participative demande beaucoup de flexibilité de la part des étudiants et du professeur: accueillir des conférenciers et faire des activités expérientielles demande parfois plus de temps que l'heure et quarante minutes allouée pour le cours (par exemple, pour faire l'activité des couvertures par $\mathrm{KAIROS}^{5}$ ).

\section{La mise en œuvre du cours}

Afin de développer des attitudes positives chez les étudiants et d'aider à renforcer les capacités en général, il devenait nécessaire de les aider à comprendre et à contextualiser l'importance de l'intégration des perspectives autochtones en éducation. Je voulais qu'ils prennent conscience en quoi cela pouvait aider à créer une société plus juste et équitable, et de leur pouvoir en tant qu'enseignants pour permettre cette transition. Je voulais inspirer les étudiants. Kanu (2011) soutient qu'en prenant conscience de notre impact, nous devenons plus résilients et persistants face aux défis potentiels. Bien qu'il ne s'agisse pas d'un cours sur les «études autochtones», je reconnaissais l'importance de graduer l'apprentissage des étudiants en les familiarisant aux événements historiques et aux défis actuels auxquels font face les collectivités autochtones et les Canadiens en général. Comme le vivais à Winnipeg et que j'interagissais avec des amis, des membres de ma famille, des collègues, des élèves, des étudiants et d'autres personnes, mon expérience personnelle m'a montré que de nombreux Canadiens ne connaissent pas les événements historiques et politiques qui ont eu des répercussions négatives sur les peuples autochtones (par exemple, beaucoup d'étudiants inscrits au cours faisant l'objet du présent article avaient peu (ou n'avaient pas) de connaissances au sujet des pensionnats, ne réalisaient pas leur 
impact sur la communauté autochtone ou ne faisaient pas de liens entre la politique d'assimilation du gouvernement fédéral à l'égard des Autochtones et certains des défis actuels - comme le sentiment de perte de l'identité, la toxicomanie, la violence, la pauvreté $)$. Cependant, j'ai remarqué que les étudiants évoluaient rapidement au fur et à mesure du déroulement des cours, ce qui est très encourageant.

J'ai conçu la séquence des cours de façon à ce que la première partie traite surtout des événements historiques essentiels à la compréhension des défis auxquels les peuples autochtones sont confrontés. Ces enseignements visaient à faciliter une com-préhension plus critique de notre réalité contemporaine collective en relation avec notre héritage colonial, en mettant en contexte les nombreux défis auxquels nous sommes confrontés aujourd'hui, et pour apprécier à quel point les communautés autochtones sont résilientes (et généreuses!). Ces histoires et ces enseignements aident les étudiants à comprendre ce que la notion de «perspectives autochtones» signifie dans un contexte éducatif, au fur et à mesure que des thèmes communs émergent des récits partagés (p. ex. l'importance des relations, le respect, la responsabilité et valorisation de la Terre). Plutôt que d'être linéaire, le processus d'apprentissage est plus cyclique. Il est important que ces histoires soient racontées à partir de diverses sources, en particulier des personnes autochtones qui ont été trop souvent oubliées. Ces personnes, dont le message est édifiant, inspirant et honnête, représentent un large éventail de voix et d'expériences de la communauté autochtone (p. ex. aînés, universitaires, travailleurs sociaux, écrivains, avocats, politiciens, pédagogues, chefs d'entreprise, activistes communautaires). Ces conférenciers invités donnent également l'occasion aux étudiants de voir des modèles de comportement autochtones positifs. Cette information donne aux étudiants une compréhension de base, celle-ci leur permettant de participer plus équitablement aux discussions et de ne pas avoir honte de se sentir ignorants. La deuxième partie du cours porte principalement sur l'intégration des perspectives autochtones dans les programmes scolaires, en termes d'approches et de contenus. Un effort a été fait pour comprendre les attentes du ministère de l'Éducation et pour explorer quelles ressources sont disponibles pour combler ces attentes (voir, par exemple, le document de l'Éducation et Formation Manitoba, 2017 qui en 
donne un excellent aperçu). Puis, nous explorons des idées sur la façon de mettre en pratique ce que les étudiants ont appris.

Pour renforcer la capacité et la confiance des enseignants en formation, il était pertinent de leur donner un aperçu des ressources pédagogiques conventionnelles officielles (ex. de la Direction des ressources éducatives françaises) et des ressources communautaires disponibles (p. ex. Commissaire des traités du Manitoba, Manitoba Metis Federation). Grâce aux conférenciers invités, aux activités en classe, aux travaux et aux visites sur le terrain, je souhaitais favoriser une interaction directe et significative par des contacts communautaires afin que les étudiants puissent intégrer ces ressources à leur propre pratique d'enseignement. Ainsi, pour favoriser un changement des attitudes et le renforcement des capacités pour surmonter l'appréhension des étudiants (Kanu, 2011), les ressources pertinentes sontessentielles (conférenciers, films, lectures, sorties communautaires, événements culturels), surtout lorsqu'elles sont soutenues par des discussions en classe. Fréquemment, ces activités et les échanges avec les conférenciers amènent les étudiants au-delà de leur zone de confort.

Pour renforcer davantage les capacités, j'ai tenté de modéliser la pédagogie que j'essayais de partager en faisant en sorte que les étudiants la vivent authentiquement en participant aux activités proposées. Le cours était expérientiel, faisait appel à la communauté, permettait le partage de récits, tentait de prendre en compte le don unique et le style d'apprentissage de chaque étudiant, ainsi que l'intégration des expériences culturelles. Les stratégies pédagogiques ont inclus: l'intégration des occasions de travail collaboratif, l'utilisation de cadres conceptuels et l'étude autonome (voir Alberta Education 2005 pour une description détaillée de ces stratégies). L'espace physique a été organisé pour être équitable et inviter à la discussion (soit des bureaux placés dans un grand cercle, parfois des groupes - ce qui était le plus approprié selon l'activité) ${ }^{7}$. Afin de créer un environnement stimulant et sécurisant où les étudiants et les invités se sentent les bienvenus et suffisamment à l'aise pour courir des risques, un temps nécessaire était alloué pour accueillir les étudiants et se renseigner sur leur bien-être. Certains protocoles appropriés ont été appris et suivis, comme la façon d'offrir du tabac aux invités autochtones en reconnaissance des connaissances partagées. 
Du thé et des pâtisseries ont été servis à chaque classe - ce partage contribuant, à mon avis, à construire une communauté d'appui. Cet environnement favorable sert à encourager chacun à se poser des questions honnêtes et difficiles qui nous amènent en tant que communauté d'apprenants à avancer sur les plans intellectuel et émotif.

\section{Les travaux}

$\mathrm{Au}$ fur et à mesure que le cours avance, les travaux sont conçus pour soutenir la progression des étudiants en tant que penseurs critiques, apprenants indépendants et futurs enseignants. Particulièrement au début, lorsque l'accent est mis sur les événements historiques et les défis actuels, le premier travail, le journal de bord, offre un espace pour réfléchir à propos de ce qui est appris par l'étudiant (Kanu, 2011). Au cours de l'année académique 2017-2018, dans un esprit d'inclusion, j'ai «ouvert» les paramètres d'expression de cette tâche afin de permettre aux étudiants de faire une réflexion personnelle sur les enjeux abordés dans le cadre du cours en proposant de faire une œuvre d'expression écrite, visuelle, ou à l'aide d'un appui sonore. Les résultats ont été extrêmement enrichissants! Pour le deuxième travail, les étudiants devaient soit participer à un événement autochtone, soit visiter un organisme ou un site autochtone; ils devaient ensuite souligner sa pertinence par rapport au cours et/ou leur futur enseignement. Cette tâche visait à encourager les rencontres positives, l'indépendance, la confiance, l'établissement de relations avec la communauté et la culture autochtone vivantes en contexte contemporain. Cette activité était suivie d'un cercle de partage où les étudiants parlaient de leurs expériences. De nombreux étudiants ont exprimé combien il était enrichissant de découvrir quelque chose de nouveau, de visiter une partie de Winnipeg dont ils ne connaissaient pratiquement rien, et combien il était positif d'avoir participé à un événement qu'ils n'auraient peut-être pas vécu autrement. Certains ayant participé à une expérience culturelle autochtone (p. ex. une hutte de sudation ou le Festival du Voyageur ${ }^{8}$ ) ont expliqué comment l'expérience avait changé leur vie (p. ex. leur sentiment de confiance en soi ou leur identité comme personne métisse). Je pense que ce travail en particulier aide les étudiants à apprendre, de façon plus honnête et positive, à propos d'une partie de notre communauté stigmatisée par les 
préjugés. Le troisième et dernier travail consistait à intégrer les perspectives autochtones (approches et contenus) dans une thématique à enseigner à un niveau scolaire au choix des étudiants. Ils devaient ensuite partager cette activité de manière significative, contextualisée et expérientielle avec le groupe. Mes observations me permettent de dire que les étudiants ont fait preuve de créativité, de sincérité et de profondeur dans la façon dont ils ont intégré les perspectives autochtones dans les activités proposées, ce qui est profondément inspirant.

\section{Considérations particulières à l'enseignement du cours en contexte francophone minoritaire au Manitoba}

Travailler dans un contexte francophone minoritaire manitobain offre certains avantages et défis particuliers. Un avantage est la lutte commune contre le processus de colonisation et d'assimilation. L'existence de la communauté métisse (souvent, à peu près d'un quart des étudiants ont des origines autochtones) témoigne de la présence d'une relation historique privilégiée entre les communautés francophone et autochtone. De plus, récemment, la communauté francophone au Manitoba a bénéficié d'une croissance importante grâce à l'immigration de personnes francophones, surtout de l'Afrique. Souvent, ces nouveaux arrivants apportent un vécu et une autre perspective internationale sur le processus de colonisation qui enrichit notre compréhension. Par conséquent, souvent en raison de cette lutte commune partagée, les étudiants francophones développent une sensibilité face aux enjeux auxquels les peuples autochtones sont confrontés.

Un défi que j’ai rencontré comme professeure dans un contexte francophone minoritaire est la question de la langue, en particulier lors de mes tentatives d'amener une présence des membres de la communauté autochtone en salle de classe ou lorsque les étudiants devaient vivre des expériences dans la communauté. En général, les étudiants sont bilingues anglaisfrançais, ce qui est un atout. Pourtant, certains des étudiants $\mathrm{n}^{\prime}$ ont pas le niveau requis en anglais pour en profiter pleinement, ce qui m’a incité à penser de façon créative à la façon de créer des ponts entre les étudiants et les membres de la communauté autochtone. Les possibilités pour les étudiants de s'engager de façon significative avec la communauté autochtone de Winnipeg sont nombreuses, mais elles se manifestent généralement en 
anglais ou encore, celles-ci sont bilingues en anglais et dans une langue autochtone (p. ex. en cri ou ojibwa). Pour surmonter cette difficulté, des ressources devaient être accessibles pour faciliter la communication (p. ex. un interprète), à la fois pour la salle de classe et pour accompagner les étudiants lorsqu'ils visitaient des organismes ou participaient à des évènements communautaires. Cependant, même avec ces ressources en place, le défi demeure celui de faciliter la compréhension, particulièrement lorsque le conférencier est un excellent conteur ou lorsque l'expérience communautaire est hautement expérientielle (p. ex. participer à une hutte de sudation). Interrompre des histoires et des enseignements pour les traduire dans ces contextes peut être gênant et potentiellement perçu comme étant impoli; la traduction simultanée est très coûteuse. Ce sont des éléments auxquels il faudrait réfléchir davantage, être créatif, et rechercher des solutions.

\section{Conclusion}

Kanu (2011) pose une question importante: «Que pouvonsnous faire en tant que pédagogues et activistes critiques pour changer ces inégalités et aider à créer une société plus juste à travers notre programme et notre pédagogie?» [traduction libre] (p. 212). En réponse à cette question, je voudrais encourager chacun d'entre nous, en particulier ceux, qui par circonstances, font que, du fait de leur naissance, peuvent choisir, ou non, d'aborder ces questions de discrimination, de ne pas avoir peur de discuter des sujets sensibles de manière honnête, informée et en offrant l'accompagnement nécessaire. Nous pouvons faire beaucoup, en particulier si nous percevons nos rôles non pas en tant qu'experts, mais en tant que facilitateurs de la connaissance. Pour ce faire, en tant que non autochtones, nous devons nous adresser à la communauté autochtone pour savoir comment nous pouvons mieux soutenir l'apprentissage et l'enseignement dans ce domaine. De plus, il serait utile de renforcer que c'est un processus d'apprentissage à vie; que, selon l'individu, ce cours universitaire peut n'être qu'un premier pas ou un autre pas dans un trajet important. Il serait bénéfique de modeler, et d'encourager les étudiants à continuer d'apprendre afin de mieux comprendre les enjeux actuels, d'apprendre à propos des contributions des Autochtones à l'histoire et dans la société contemporaine. 
Je me demande souvent pourquoi ce cours, jusqu'ici, a été si bien reçu. En général, j'ai observé que les étudiants étaient inspirés et motivés à venir en classe. Dans les évaluations de cours, les étudiants ont expliqué qu'ils trouvaient les différents thèmes intéressants, et qu'ils en sont venus à mieux comprendre la relation entre les événements historiques et les réalités actuelles dont ils sont témoins. Ils ont apprécié que les discussions aient été riches et sans jugement, et qu'un lien clair ait été établi avec la pratique éducative. C'est difficile à décrire, mais c'est comme s'il y avait une véritable honnêteté dans le cours, c'est comme si nous faisions partie de quelque chose de plus grand. Je sens que nous arrivons finalement à comprendre une partie de notre réalité d'une manière beaucoup plus éclairée, comme si un linceul avait été retiré d'une partie de notre expérience collective canadienne et que ce processus d'illumination avait été profondément émouvant pour nous tous. Personnellement, enseigner ce cours a transformé, doucement, la perception que j'avais de moi-même, ce que cela représente d'être d'ici (c.-à.-d., ce que cela représente d'être originaire de la vallée de la Rivière Rouge, de Winnipeg, des prairies, du Manitoba, du Canada...), et mes responsabilités comme citoyenne face à la collectivité et à la Terre. Plus j'apprends, mieux je comprends ce que veut dire vivre l'esprit des traités et de la réconciliation. Comme le soulève Kanu (2011), ce cours, plus que tout autre que j'ai enseigné, est un cours d'étude et un voyage émotionnel dans l'enseignement et l'apprentissage, (c.-à-d. currere).

\section{NOTES}

1. Cadres fondamentaux indigènes/autochtones pour le développement des enseignants et enseignantes - la perspective manitobaine (les 4 et 7 juin 2013). Une communication présentée dans le cadre du colloque de la Société canadienne pour l'étude de l'éducation tenu à l'Université de Victoria et dans le cadre du colloque du MERN - Treaties and Reconciliation Forum à l'Université de Brandon. Cette communication a été donnée en collaboration avec David Anderson et Sherry Peden, Collège universitaire du Nord; Marlene Atleo, Frank Deer, Laara Fitznor, Université du Manitoba; et Kevin Lamoureux, Université de Winnipeg.

2. Les «contre-histoires» font référence à des récits ou explications alternatifs ou opposés, souvent à des courants dominants, hégémoniques ou dominants. 
3. Voir https://www.micec.com pour plus d'information sur les services qu'ils offrent.

4. Point Douglas fait partie du «North End» de Winnipeg. Historiquement, elle est une des plus vieilles communautés de la ville. Actuellement, c'est un quartier qui contient un des codes postaux où l'on retrouve les populations les plus démunies au Canada. Cette communauté est connue pour sa population à prédominance autochtone (Comack et al., 2009; Kives, 2014).

5. Le programme KAIROS Activité des couvertures (Blanket exercise) «est une leçon d'histoire participative unique, développée en collaboration avec des aînés autochtones, des gardiens du savoir et des pédagogues, qui favorise la vérité, la compréhension, le respect et la réconciliation entre peuples autochtones et non autochtones.» [traduction libre] Description repérée du https:// www.kairosblanketexercise.org/

6. Ces enjeux sont explorés par des auteurs comme Comack et al. $(2009,2013)$ et Sinclair (2016) entre autres.

7. Fait intéressant, des rénovations récentes $m^{\prime}$ ont fait prendre conscience de la façon dont les espaces physiques permettent ou contraignent la communication entre nous. D'après ce quej'observe dans une salle de classe en particulier, les rénovations récentes ont été conçues autour de l'utilisation de la technologie, avec des prises électriques pour chaque siège. Face à l'avant, les tables sont disposées en rangées, les câbles électriques reliant les tables les unes aux autres et au mur, ce qui rend difficile la réorganisation de cet espace physique, qui renforce une structure hiérarchique où l'enseignant est au centre de l'apprentissage; s'asseoir dans un cercle est presque impossible. Cela met en évidence la façon dont une configuration physique en contexte éducatif peut essentiellement rendre impossible l'enseignement par d'autres moyens, tels que l'intégration des pédagogies autochtones (Beeman et Sims, sous presse).

8. Le Festival du Voyageur est un festival d'hiver qui célèbre le passé canadien de la traite des fourrures ainsi que le patrimoine et la culture francophone et métisse. Voir le site web du Festival https:// www.heho.ca/fr/

\section{BIBLIOGRAPHIE}

ALBERTA EDUCATION (2005) Nos mots, nos façons: enseigner aux apprenants des Premières nations, des Métis et des Inuits. Edmonton, Aboriginal Services Branch, 198 p.

ANDERSON, Doug, COMAY, Julie, et CHIARIOTTO, Lorraine (2017) Curiosité naturelle, $2^{e}$ édition: ressource pour l'enseignante ou l'enseignant. L'importance du point de vue autochtone dans 
l'enquête dans l'environnement de l'enfant. École-laboratoire du Dr. Eric Jackman Institute of Child Study. Institut d'études pédagogiques de l'Ontario, Université de Toronto. Toronto: Marquis Imprimeur. 342 p. Repéré à www.naturalcuriosity. ca

ASSOCIATION CANADIENNE DES DOYENS ET DOYENNES D'ÉDUCATION (2010). Accord sur l'éducation autochtone. 8 p. Repéré à www.csse.ca/ACDE

BEEMAN, Chris, et SIMS, Laura (sous presse) «From relationship to something more: Environmental and sustainability education and a new ontological position», dans KARROW, Doug, DIGIUSEPPE, Maurice, BELL, Nicole, ELLIOT, Paul, et INWOOD, Hilary (dir.) Environmental and sustainability education in preservice teacher education: Proceedings of Canadian national roundtable 2016, Springer Canada.

BLOCK, Lee Ann, SIMS, Laura et BEEMAN, Chris (2016) «Contextualizing education for sustainability and teacher education in Manitoba faculties of education», dans KARROW, Doug, DIGIUSEPPE, Maurice, ELLIOTT, Paul, GWEKWERERE, Yovita, et INWOOD, Hilary (dir.) Canadian perspectives on initial teacher environmental education praxis, Ottawa, Canadian Association for Teacher Education (CATE), p. 128-152.

COMACK, Elizabeth, DEANE, Lawrence, MORISSETTE, Larry, et SILVER, Jim (2009) Pour agir sur la violence du quartier, il faut agir sur le quartier: Violence et gangs de rue au centreville de Winnipeg, Winnipeg, Centre canadien de politiques alternatives, $14 \mathrm{p}$.

(2013). Indians wear red: Colonialism, resistance, and Aboriginal street gangs, Winnipeg, Fernwood, $166 \mathrm{p}$.

DEER, Frank (2013) «Integrating aboriginal perspective in education: Perceptions of pre-service teachers», Canadian journal of education, vol. 36, $\mathrm{n}^{\circ} 2$, p. 175-211.

ÉDUCATION ET FORMATION MANITOBA (2017) L'intégration des perspectives autochtones dans le milieu scolaire de langue française: une approche pédagogique inspirée par les visions $d u$ monde autochtones. Repéré à http://www.edu.gov.mb.ca/m12/ frpub/ped/autochtones/perspectives/docs/doc_complet.pdf

GOUVERNEMENT DU MANITOBA (2007) Aboriginal education directorate. Repéré à http://www.edu.gov.mb.ca/aed/index. html 
KANU, Yatta (2005) «Teachers' perceptions of the integration of Aboriginal culture into the high school curriculum», The Alberta journal of educational research, vol. 51, nº 1, p. 50-68.

(2011) Integrating Aboriginal perspectives into the school curriculum: Purposes, possibilities, and challenges. Toronto, University of Toronto Press, $244 \mathrm{p}$.

KIVES, Bartley (2014, 21 octobre) «The 'great indigenous divide': Winnipeg stares into the ethnic chasm». The guardian international edition. Repéré à https://www.theguardian. com/cities/2014/oct/21/winnipeg-election-indigenous-divideaboriginal

MACDONALD, Nancy (2015, 22 janvier) «Welcome to Winnipeg: Where Canada's racism problem is at its worst». Maclean's. Reperé à http://www.macleans.ca/news/canada/welcome-towinnipeg-where-canadas-racism-problem-is-at-its-worst/.

MANITOBA ASSOCIATION OF SCHOOL SUPERINTENDENTS (2013) Transforming Manitoba public education: A view to the future. MASS position statement on Aboriginal education. Repéré à http://mass.mb.ca/wp-content/ uploads/2015/01/transforming_mb_public_education 2013. pdf

SIMS, Laura (2012) «Taking a learning approach to community-based strategic environmental assessment: Results from a Costa Rican case study», Impact assessment and project appraisal, vol. 30, n 4, p. 242-252.

(2015) «Inspired to face the hurdles: A non-indigenous educator's experience facilitating the integration of Aboriginal perspectives into education». MERN occasional paper series: Indigenous education No. 1. Reperé à http://mbtrc.org/data/ documents/occ-1.pdf.

(2017) «Learning for sustainability through CIDA's “Communitybased pest management in Central American agriculture project": A deliberative, experiential and iterative process», Journal of environmental planning and management, vol. 60, $\mathrm{n}^{\circ} 3$, p. 538-557. doi:10.1080/09640568.2016.1165188.

SIMS, Laura et FALKENBERG, Thomas (2013) «Developing competencies for education for sustainable development: A case study of Canadian faculties of education». International journal of higher education, vol. 2, n 4, p. 1-14.

SIMS, Laura et SINCLAIR, Alan John (2008) «Learning through participatory resource management programs: Case studies from Costa Rica». Adult education quarterly, vol. 58, $\mathrm{n}^{\circ} 2$, p. 151-168. 
SINCL AIR, Niigaan (2016) «Reconciliation lives here: The 2016 state of the inner city report», dans State of the inner city: Reconciliation lives here, Winnipeg, Manitoba: Centre canadien de politiques alternatives, p. 1-4.

ST.-DENIS, Verna (2007) «Aboriginal education and anti-racist education: Building alliances across cultural and racial identity». Canadian journal of education, vol. 30, n 4, p. 1068-1092. 